Discrete Comput Geom 29:505-510 (2003)

DOI: $10.1007 /$ s00454-003-0784-9

\title{
Tverberg's Conjecture*
}

\author{
Siniša T. Vrećica \\ Faculty of Mathematics, University of Belgrade, \\ Studentski trg 16, P.O.B. 550, 11000 Belgrade, Serbia \\ vrecica@matf.bg.ac.yu
}

\begin{abstract}
In 1989 Helge Tverberg proposed a quite general conjecture in Discrete Geometry, which could be considered as the common basis for many results in Combinatorial Geometry, and at the same time as a discrete analogue of the common transversal theorems. It implies or contains as special cases many classical "coincidence" results such as Radon's theorem, Rado's theorem, the Ham sandwich theorem, "non-embeddability" results (e.g. non-embeddability of graphs $K_{5}$ and $K_{3,3}$ in $\mathbb{R}^{2}$ ), etc. The main goal of this short note is to verify this conjecture in one new, non-trivial case. We obtain the continuous version of the conjecture. So, it is not surprising that we use topological methods, or more precisely the methods of equivariant topology and the theory of characteristic classes.
\end{abstract}

\section{Introduction}

Establishing the relation between Rado's theorem on general measure (see [11]) and the Ham sandwich theorem, the following result is proved in [21] stating that these two results belong to the same family.

Theorem 1.1. Let $0 \leq k \leq d-1$, and let $\mu_{0}, \mu_{1}, \ldots, \mu_{k}$ be $\sigma$-additive probability measures on $\mathbb{R}^{d}$. Then there is a $k$-flat $F$ with the property that every closed halfspace containing $F$, has $\mu_{i}$-measure at least $1 /(d-k+1)$ for all $i, 0 \leq i \leq k$.

Namely, this theorem reduces to Rado's theorem in the case $k=0$ and to the Ham sandwich theorem in the case $k=d-1$.

The proof of the above theorem uses the topological result claiming the non-existence of a non-zero section of a certain vector bundle over the Grassmann manifold. Helge

* This research was supported by the Ministry for Science, Technology and Development of Serbia, Grant 1854. 
Tverberg observed that the special case $k=0$ (Rado's theorem) follows easily from his result in Combinatorial Geometry from [13].

Theorem 1.2. Let $S$ be a set of $(r-1)(d+1)+1$ points in $\mathbb{R}^{d}$. Then one can split it into subsets $S_{1}, S_{2}, \ldots, S_{r}$ so that

$$
\bigcap_{i=1}^{r} \operatorname{conv} S_{i} \neq \emptyset
$$

This observation motivated him to suppose that a general result should exist which would generalize his result, Theorem 1.2, and at the same time it would imply Theorem 1.1 in the same way as his result implied Rado's theorem. So, he formulated the following Tverberg's conjecture:

Conjecture 1.3. Let $0 \leq k \leq d-1$ and let $S_{0}, S_{1}, \ldots, S_{k}$ be finite sets of points in $\mathbb{R}^{d}$, with $\left|S_{i}\right|=\left(r_{i}-1\right)(d-k+1)+1$ for $i=0,1, \ldots, k$. Then $S_{i}$ can be split into $r_{i}$ sets, $S_{i 1}, S_{i 2}, \ldots, S_{i r_{i}}$, so that there is a $k$-flat $F$ meeting all the sets conv $S_{i j}, 0 \leq i \leq$ $k, 1 \leq j \leq r_{i}$.

It is easy to see that this conjecture implies Theorem 1.1 ([14], see also [20]) and its special case $k=0$ is Theorem 1.2. This conjecture unifies two important themes of Combinatorial Geometry: Helly-type theorems (the special case $r=2$ of Theorem 1.2 is the well-known Radon's theorem), and the common transversal theorems. Moreover, it was shown in [20] that this result could be considered as an example of the whole family of results of "combinatorial geometry on vector bundles", and that these results would also generalize many coincidence results such as the non-embeddability of the graphs having minor $K_{5}$ or $K_{3,3}$ in the plane, etc.

Both Theorem 1.2 and Conjecture 1.3 could be reformulated in terms of linear mappings and then generalized to the continuous case. Let us consider, for each $i=$ $0,1, \ldots, k$, the $N_{i}$-dimensional simplex $\Delta_{i}^{N_{i}}$ with the vertices $e_{0}^{i}, e_{1}^{i}, \ldots, e_{N_{i}}^{i}$, where $N_{i}=\left(r_{i}-1\right)(d-k+1)$, and also the linear mapping $\varphi_{i}: \Delta_{i}^{N_{i}} \rightarrow \mathbb{R}^{d}$ sending the vertices of $\Delta_{i}^{N_{i}}$ to the points of the set $S_{i}$. We notice that the convex hull of some subset of $S_{i}$ is the $\varphi_{i}$-image of the appropriate face of the simplex $\Delta_{i}^{N_{i}}$. If we replace the linear mappings by the continuous mappings, we obtain the continuous version of Tverberg's conjecture.

Conjecture 1.4. Let $0 \leq k \leq d-1$ and let $\varphi_{i}: \Delta_{i}^{N_{i}} \rightarrow \mathbb{R}^{d}$ for $i=0,1, \ldots, k$ be continuous mappings. Then there is an affine $k$-flat $F$ which intersects the images of $r_{i}$ pairwise disjoint faces of simplex $\Delta_{i}$ for each $i=0,1, \ldots, k$.

The special case $k=0$ of this conjecture generalizes Theorem 1.2 and is known as the continuous Tverberg theorem. It is established for $r=2$ by Bajmóczy and Bárány in [1] and when $r$ is an odd prime number by Bárány et al. in [3]. When $r$ is a power of a prime number, this is done by Özaydin in [10] and later also by Volovikov in [16], Sarkaria in [12] and de Longueville in [6]. 
In [15] this conjecture was verified in some special cases. It was proved that, besides the already known case $k=0$, the conjecture is true in the case $k=d-1$, and in the case $k=1$ when $r_{0}=1$ or $r_{1}=1$ or $r_{0}=r_{1}=2$. Also, a slightly weakened version of the conjecture is proved in the case $k=d-2$, obtained when $3 r_{i}$ points in $S_{i}$ were considered instead of $3 r_{i}-2$ of them. This version still suffices to imply Theorem 1.1 in the case $k=d-2$.

A much more general result verifying some cases of this conjecture was achieved by Živaljević in [20], where he established Conjecture 1.3 when $r_{0}=r_{1}=\cdots=r_{k}$ is an odd prime number, and both $d$ and $k$ are odd integers.

The main result of this paper is establishing Conjecture 1.3 in the case $r_{0}=r_{1}=$ $\cdots=r_{k}=2$ without any additional restriction.

The result in [20] is obtained by using parametrized, ideal-valued, cohomological index theory. The method of this paper will again be the reduction of the result to the non-existence of some equivariant mapping, or equivalently to the non-existence of the section of certain sphere bundle. This will be proved by showing that the corresponding Stiefel-Whitney class is non-trivial. For the theory of characteristic classes consult [9].

Although Conjecture 1.3 is a statement in Combinatorial Geometry, all the results establishing some of its special cases, establish its more general, continuous version (Conjecture 1.4) and use topological methods. For very nice accounts on the topological methods in Combinatorics and Combinatorial Geometry see [2], [4], [18], [17], [19] and [8].

\section{The Result}

As we already mentioned, the main goal of this paper is to establish Conjecture 1.3 in the case $r_{0}=r_{1}=\cdots=r_{k}=2$, i.e. to prove the following theorem.

Theorem 2.1. Let $0 \leq k \leq d-1$ and let $S_{0}, S_{1}, \ldots, S_{k}$ be finite sets, each of $d-k+2$ points in $\mathbb{R}^{d}$. Then every set $S_{i}$ can be split into two sets, $S_{i 1}$ and $S_{i 2}$, so that there is a $k$-flat $F$ meeting all the sets conv $S_{i j}, 0 \leq i \leq k, j \in\{1,2\}$.

More generally, we will prove the continuous version of this statement, i.e. the case $r_{0}=r_{1}=\cdots=r_{k}=2$ of Conjecture 1.4. We consider the family of $(d-k+1)$ dimensional simplices $\Delta_{0}, \Delta_{1}, \ldots, \Delta_{k}$, where $\Delta_{i}$ is the simplex spanned by the vertices $e_{0}^{i}, e_{1}^{i}, \ldots, e_{d-k+1}^{i}$ for $i=0,1, \ldots, k$.

Theorem 2.2. Let $0 \leq k \leq d-1$ and let $\varphi_{i}: \Delta_{i} \rightarrow \mathbb{R}^{d}$ for $i=0,1, \ldots, k$ be continuous mappings. Then there is an affine $k$-flat $F$ which intersects the images of two disjoint faces of every simplex $\Delta_{i}$.

Proof. We denote with $\mathrm{Gr}_{d, d-k}$ the Grassmann manifold of $(d-k)$-dimensional linear subspaces of $\mathbb{R}^{d}$, and, for any $L \in \mathrm{Gr}_{d, d-k}$, with $\pi_{L}: \mathbb{R}^{d} \rightarrow L$ the orthogonal projection.

We consider the $(d-k+1)$-dimensional simplices $\Delta_{i}=\operatorname{conv}\left\{e_{0}^{i}, e_{1}^{i}, \ldots, e_{d-k+1}^{i}\right\}$, $i=0,1, \ldots, k$. With $\left(\Delta_{i}\right)_{*}^{2}$ we denote the deleted square of the simplex $\Delta_{i}$, i.e. the set of ordered pairs of points in $\Delta_{i}$ having disjoint supports. (A support of a point is a face 
of the simplex containing it in its interior.) It is easy to verify that the deleted square $\left(\Delta_{i}\right)_{*}^{2}$ is a $(d-k)$-dimensional manifold which we denote by $M$. It could be proved that $M$ is actually homeomorphic to the sphere $S^{d-k}$, but we do not need this fact here. (It is shown in [3] that $M$ is $(d-k-1)$-connected.)

The statement of the theorem reduces to the claim that there exists $L \in \mathrm{Gr}_{d, d-k}$ such that

$$
\pi_{L}\left(\varphi_{0}\left(x_{0}\right)\right)=\pi_{L}\left(\varphi_{0}\left(y_{0}\right)\right)=\cdots=\pi_{L}\left(\varphi_{k}\left(x_{k}\right)\right)=\pi_{L}\left(\varphi_{k}\left(y_{k}\right)\right)
$$

for some $\left(\left(x_{0}, y_{0}\right), \ldots,\left(x_{k}, y_{k}\right)\right) \in\left(\Delta_{0}\right)_{*}^{2} \times \cdots \times\left(\Delta_{k}\right)_{*}^{2}$. Here $L=F^{\perp}$, i.e. $L$ is the orthogonal complement to the affine $k$-flat $F$ claimed to exist in the statement of the theorem.

We denote with $\xi$ the canonical vector bundle over $\mathrm{Gr}_{d, d-k}$. For every $L \in \mathrm{Gr}_{d, d-k}$, we have the mapping

$$
\begin{gathered}
\psi_{L}:\left(\Delta_{0}\right)_{*}^{2} \times \cdots \times\left(\Delta_{k}\right)_{*}^{2} \rightarrow L^{2 k+2}, \\
\psi_{L}\left(\left(x_{0}, y_{0}\right), \ldots,\left(x_{k}, y_{k}\right)\right)=\left(\pi_{L}\left(\varphi_{0}\left(x_{0}\right)\right), \pi_{L}\left(\varphi_{0}\left(y_{0}\right)\right), \ldots, \pi_{L}\left(\varphi_{k}\left(x_{k}\right)\right), \pi_{L}\left(\varphi_{k}\left(y_{k}\right)\right)\right) .
\end{gathered}
$$

The group $G=\underbrace{\mathbb{Z} / 2 \oplus \cdots \mathbb{Z} / 2}_{k+1}$ acts on these spaces, freely on $\left(\Delta_{0}\right)_{*}^{2} \times \cdots \times\left(\Delta_{k}\right)_{*}^{2}$ $\approx M^{k+1}$ and fiberwise on $\xi^{2 k+2}$ (i.e. trivially on $\mathrm{Gr}_{d, d-k}$ and the $i$ th generator of $G$ interchanges the $(2 i-1)$ th and $(2 i)$ th coordinate of the fiber). The above mapping is equivariant and it induces a section $s$ of the vector bundle

$$
\xi^{2 k+2} \times{ }_{G} M^{k+1} \rightarrow\left(\mathrm{Gr}_{d, d-k} \times M^{k+1}\right) / G=\mathrm{Gr}_{d, d-k} \times M^{k+1} / G
$$

The fiber over $\left[L,\left(x_{0}, y_{0}, \ldots, x_{k}, y_{k}\right)\right]$ could be identified with $L^{2 k+2}$. The statement of the theorem now reduces to the claim that the section $s$ intersects the diagonal $\Delta$ in some fiber. Let us suppose, to the contrary, that $s$ does not intersect the diagonal in any fiber. Projecting to the orthogonal complement of the diagonal and then radially to its sphere (in each fiber), we obtain the non-zero section of the vector bundle with the fiber $L^{2 k+1}$ and the section of the associated sphere bundle whose fiber is homeomorphic with $S^{(d-k)(2 k+1)-1}$.

We reach a contradiction (proving in this way the theorem) by showing that the topdimensional Stiefel-Whitney class of this sphere bundle does not vanish. The Poincaré dual of the top-dimensional Stiefel-Whitney class of the sphere bundle coincides with the homology class of the zero-set of a section of the associated vector bundle (with the fiber $L^{2 k+1}$ ), which is transversal to the zero section. (For example, see [7] and also Theorem 11.17 of [5], in the oriented case.) So, it suffices to find a section of the corresponding vector bundle which intersects the zero section transversally in an odd number of points.

In order to construct such a section we consider $k+1$ parallel $(d-k)$-dimensional affine planes $A_{0}, A_{1}, \ldots, A_{k}$ in $\mathbb{R}^{d}$ at distance $m$ one from each other, and $d-k+2$ points in each of them being the vertices $v_{0}^{i}, v_{1}^{i}, \ldots, v_{d-k}^{i}$ and the barycenter $\hat{\sigma}_{i}$ of a $(d-k)$ dimensional simplex $\sigma_{i}$, of diameter $M, 0 \leq i \leq k$. Let us suppose that they are in a generic position meaning that their $k+1$ barycenters span an affine $k$-dimensional flat $F$. (Let us also choose them so that the flat $F$ is orthogonal to the planes $A_{0}, A_{1}, \ldots, A_{k}$.) 
We also consider linear mappings $f_{i}$ which map vertices of $\Delta_{i}$ to the vertices and the barycenter of $\sigma_{i}$, namely, $f_{i}\left(e_{j}^{i}\right)=v_{j}^{i}, 0 \leq j \leq d-k$ and $f_{i}\left(e_{d-k+1}^{i}\right)=\hat{\sigma}_{i}$ for $i=0,1, \ldots, k$.

The images under the linear mapping $f_{i}$ of two disjoint faces of the simplex $\Delta_{i}$ are the convex hulls of the corresponding vertices of the simplex $\sigma_{i}$ and its barycenter $\hat{\sigma}_{i}$. The only non-empty intersection of the convex hulls of two disjoint subsets of $\left\{v_{0}^{i}, v_{1}^{i}, \ldots, v_{d-k}^{i}, \hat{\sigma}_{i}\right\}$ is

$$
\operatorname{conv}\left\{v_{0}^{i}, v_{1}^{i}, \ldots, v_{d-k}^{i}\right\} \cap \operatorname{conv}\left\{\hat{\sigma}_{i}\right\}=\left\{\hat{\sigma}_{i}\right\}
$$

If some affine $k$-dimensional plane in $\mathbb{R}^{d}$ intersects the images of two disjoint faces of some simplex $\Delta_{i}$, then this plane contains the barycenter $\hat{\sigma}_{i}$ or it intersects $A_{i}$ in at least the one-dimensional affine plane. If this plane should intersect the images of two disjoint faces of each simplex $\Delta_{0}, \Delta_{1}, \ldots, \Delta_{k}$, then (because of the generic position of the planes $A_{0}, A_{1}, \ldots, A_{k}$ ) it has to contain the barycenters $\hat{\sigma_{0}}, \hat{\sigma_{1}}, \ldots, \hat{\sigma_{k}}$ and it is uniquely determined by them. So, the mappings $f_{0}, f_{1}, \ldots, f_{k}$ induce the section of the considered vector bundle which intersects the diagonal at a single orbit of the action of the group $G$, i.e. at the orbit

$$
\left[L,\left(\frac{e_{0}^{0}+\cdots+e_{d-k}^{0}}{d-k+1}, e_{d-k+1}^{0}, \ldots, \frac{e_{0}^{k}+\cdots+e_{d-k}^{k}}{d-k+1}, e_{d-k+1}^{k}\right)\right],
$$

where $L=F^{\perp}$ is the orthogonal complement to the $k$-dimensional flat $F$ spanned by the points $\hat{\sigma_{0}}, \hat{\sigma_{1}}, \ldots, \hat{\sigma_{k}}$.

So, we found a section which intersects the zero section of the considered vector bundle in a single point. For small perturbation of the mappings $f_{i}$, the intersection with the zero section will remain a single point. Namely, it could be easily seen that for continuous mappings $g_{i}$ sufficiently close to the mappings $f_{i}$ (let us say $\left\|f_{i}-g_{i}\right\|<\varepsilon$ ), the only $k$-dimensional plane intersecting the $g_{i}$-images of two disjoint faces of the simplices $\Delta_{i}$ is again the plane spanned by the images of the barycenters, i.e. the points $g_{0}\left(e_{d-k+1}^{0}\right), \ldots, g_{k}\left(e_{d-k+1}^{k}\right)$. If some $k$-dimensional plane $F^{\prime}$ did not contain the image of some barycenter $g_{i}\left(e_{d-k+1}^{i}\right)$, it would have to contain two different points (at the distance bounded below by $l-2 \varepsilon$, where $l$ is the minimal distance of the barycenter of some simplex to its closest face) which are both very close to the plane $A_{i}$. This would make the plane $F^{\prime}$ contain a line (determined by those two points) very close to the plane $A_{i}$ (meaning that the angle $\alpha$ among that line and the plane $A_{i}$ could be as small as necessary, i.e. $\tan \alpha \leq 2 \varepsilon /(l-2 \varepsilon))$. Then the plane $F^{\prime}$ could not intersect the images of all simplices. It would be enough to choose $\varepsilon$ to be much smaller than $(m \cdot l) / 4 M$. Namely, if $F^{\prime}$ intersected the images of all simplices, then the angle $\beta$ among any line contained in $F^{\prime}$ and the plane parallel with $A_{i}$ would not be small, i.e. $\tan \beta \geq(m-2 \varepsilon) /(2 M+2 \varepsilon)$, which is not true for the line considered above because $\varepsilon$ is chosen to be much smaller than $(m \cdot l) / 4 M$. So, the obtained section intersects the zero section transversally. This completes our proof. 


\section{Remarks}

Since our Theorem 2.2 establishes the continuous version of the conjecture in the case $r_{0}=r_{1}=\cdots=r_{k}=2$, it generalizes not only Tveberg's theorem, but also the result of Bajmóczy and Bárány from [1] in the same way as the result from [20] generalizes the result of Bárány et al. from [3].

Our method does not use the fact that $r=2$ is a prime, which might lead to the hope that it could be used to establish the conjecture (or at least its special case $k=0$ ) for non-prime $r$. Unfortunately, it does not work for $r>2$. Namely, in that case the constructed section of the given vector bundle intersects the zero section in an even number of points. Also, the configuration space corresponding to $M^{k+1}$ in that case is not a manifold, and so Poincaré duality could not be used.

\section{References}

1. E.G. Bajmóczy and I. Bárány, On a common generalization of Borsuk's and Radon's theorem, Acta Math. Acad. Sci. Hungar. 34 (1979), 347-350.

2. I. Bárány, Geometric and combinatorial applications of Borsuk's theorem, in New Trends in Discrete and Computational Geometry, János Pach, ed., Algorithms and Combinatorics 10, Springer-Verlag, Berlin, 1993, pp. 235-249.

3. I. Bárány, S.B. Shlosman and A. Szücs, On a topological generalization of a theorem of Tverberg, J. London Math. Soc. 23 (1981), 158-164.

4. A. Björner, Topological methods, in Handbook of Combinatorics, R. Graham, M. Grötschel and L. Lovász, eds., North-Holland, Amsterdam, 1995, pp. 1819-1872.

5. R. Bott and L.W. Tu, Differential Forms in Algebraic Topology, Springer-Verlag, New York, 1982.

6. M. de Longueville, Notes on the topological Tverberg theorem, Discrete Math. 241(1-3) (2001), 207-233.

7. D.B. Fuchs and O.Y. Viro, Topology II (Homotopy and Homology. Classical Manifolds), Springer-Verlag, Berlin, 2003.

8. J. Matoušek, Using the Borsuk-Ulam Theorem, Lectures on Topological Methods in Combinatorics and Geometry (in preparation).

9. J. Milnor and J. Stasheff, Characteristic Classes, Princeton University Press, Princeton, NJ, 1974.

10. M. Özaydin, Equivariant maps for the symmetric group, Unpublished manuscript, 1987.

11. R. Rado, A theorem on general measure, J. London Math. Soc. 21 (1946), 291-300.

12. K. Sarkaria, Tverberg's partitions and Borsuk-Ulam theorems, Pacific J. Math. 196 (2000), 231-241

13. H. Tverberg, A generalization of Radon's theorem, J. London Math. Soc. 41 (1966), 123-128.

14. H. Tverberg, A talk at the Symposium on Combinatorics and Geometry, Stockholm, 1989.

15. H. Tverberg and S.T. Vrećica, On generalizations of Radon's theorem and the ham sandwich theorem, European J. Combin. 14 (1993), 259-264.

16. A.Yu. Volovikov, On a topological generalization of the Tverberg theorem, Math. Notes 59(3) (1996), 324-326. (Translation from Mat. Zametki 59(3) (1996), 454-456.

17. R.T. Živaljević, User's guide to equivariant methods in combinatorics, Publ. Inst. Math. Belgrade, 59(73) (1996), 114-130

18. R.T. Živaljević, Topological methods, in CRC Handbook of Discrete and Computational Geometry, J.E. Goodman and J. O'Rourke, eds., CRC Press, Boca Raton, FL, 1997, pp. 209-224.

19. R.T. Živaljević, User's guide to equivariant methods in combinatorics, II, Publ. Inst. Math. Belgrade, 64(78) (1998), 107-132.

20. R.T. Živaljević, The Tverberg-Vrećica problem and the combinatorial geometry on vector bundles, Israel J. Math. 111 (1999), 53-76.

21. R.T. Živaljević and S.T. Vrećica, An extension of the ham sandwich theorem, Bull. London Math. Soc. 22 (1990), 183-186.

Received April 24, 2002, and in revised form December 2, 2002. Online publication March 28, 2003. 